\title{
Epithelial folliculin is involved in airway inflammation in workers exposed to toluene diisocyanate
}

\author{
Duy L Pham ${ }^{1,2,3,4}$, Tu HK Trinh ${ }^{1,4}$, Ga-Young Ban ${ }^{1}$, Seung-Hyun Kim ${ }^{1}$ and Hae-Sim Park ${ }^{1,2}$
}

Toluene diisocyanate (TDI) exposure can directly activate and damage airway epithelium. Folliculin (FLCN) is a protein expressed by human airway epithelial cells (HAECs) to maintain airway epithelial integrity and survival. This study investigated the involvement of FLCN in the pathogenesis of TDI-induced occupational asthma (OA). Enzyme-linked immunosorbent assay was used to measure serum levels of FLCN in TDI-exposed subjects (93 TDI-OA patients and 119 asymptomatic exposed controls (AEC)), 200 non-occupational asthma (NOA) patients and 71 unexposed healthy normal controls (NCs). Significantly more subjects in the TDI-OA and AEC groups had high serum levels of FLCN compared to those in the NOA group $(P=0.002$ and $P=0.001$, respectively), all of which were higher than the NC group (all $P<0.001$ ). The serum level of FLCN was positively correlated with TDI exposure duration ( $r=0.251, P=0.027$ ), but was negatively correlated with asthma duration of TDI-OA patients $(r=-0.329, P=0.029)$. TDI-exposed subjects with high FLCN levels had higher serum levels of total IgE than those with lower levels. The effects of TDI exposure on FLCN production was investigated by treating HAECs (A549 cells) with TDIhuman serum albumin conjugate, which showed increased expression and release of FLCN and interleukin-8 from HAECs. Coculture with peripheral blood neutrophils also induced FLCN expression and release from HAECs. In conclusion, TDI exposure and TDI-induced neutrophil recruitment into the airways can activate and stimulate HAECs to produce FLCN, which could be involved in airway inflammation in workers exposed to TDI.

Experimental \& Molecular Medicine (2017) 49, e395; doi:10.1038/emm.2017.180; published online 17 November 2017

\section{INTRODUCTION}

Toluene diisocyanate (TDI) is a low molecular weight compound used in the production of polyurethane foams, automobile paint, varnishes and related products. Long-term exposure to TDI can lead to the development of TDIinduced occupational asthma (OA), a common OA in industrialized countries worldwide. ${ }^{1}$ TDI-OA has a poor prognosis and its pathogenesis is not completely understood, but could be more complicated than that of non-occupational asthma (NOA). Exposure to TDI directly damages airway epithelium, leading to increased airway epithelial permeability. Moreover, TDI exposure activates airway epithelial cells, which produce cytokines and recruit inflammatory cells such as neutrophils into the airways, which then enhances the airway inflammation and leads to a severe asthma phenotype.,3

Folliculin (FLCN) is expressed in the skin, kidneys, and upper and lower airway epithelial cells. ${ }^{4}$ Mutations in the FLCN gene have been implicated in Birt-Hogg-Dube syndrome, which is characterized by lung cysts and spontaneous pneumothorax. ${ }^{5,6}$ Recent studies have reported the role of FLCN in maintaining the integrity and function of airway epithelial cells and lung fibroblasts. Knockout of FLCN induced apoptosis, downregulated the expression of E-cadherin in mouse lung alveolar cells and damaged several signaling pathways in human airway epithelial cells (HAECs). ${ }^{7,8}$ These findings suggest that FLCN may involve in chronic airway diseases that involve airway damage. However, to date, the involvement of FLCN in OA and NOA is unknown.

In this study, we measured the serum levels of FLCN in patients with TDI-OA, asymptomatic TDI-exposed controls (AECs), NOA patients and unexposed normal healthy controls (NCs) to evaluate the involvement of FLCN in these diseases. We also investigated the effects of TDI and neutrophil inflammation on HAEC activation and their production of FLCN.

\footnotetext{
${ }^{1}$ Department of Allergy and Clinical Immunology, Ajou University School of Medicine, Suwon, South Korea; ${ }^{2}$ Department of Biomedical Sciences, The Graduate School, Ajou University, Suwon, South Korea and ${ }^{3}$ Department of Physiology, Faculty of Medicine, University of Medicine and Pharmacy, Ho Chi Minh City, Vietnam

${ }^{4}$ These authors contributed equally to this work.

Correspondence: Professor H-S Park, Department of Allergy and Clinical Immunology, Ajou University School of Medicine, San-5, Worldcup-road 164, Youngtong-gu, Suwon 443-380, South Korea.

E-mail: hspark@ajou.ac.kr

Received 27 January 2017; revised 18 April 2017; accepted 8 May 2017
} 


\section{MATERIALS AND METHODS}

\section{Subject recruitment}

We recruited 212 TDI-exposed workers (including 93 TDI-OA patients and 119 AECs), 200 NOA patients and 71 NCs from Ajou University Medical Center (Suwon, South Korea). TDI-OA was diagnosed by a positive response to a TDI bronchoprovocation test, as previously described. ${ }^{9}$ A diagnosis of Birt-Hogg-Dube syndrome was excluded based on disease history and chest X-ray. One week before serum collection, the TDI-OA and NOA patients stopped using leukotriene modifiers and anti-inflammatory agents, including inhaled or oral corticosteroid. Serum samples were collected at the time of diagnosis and stored at $-70{ }^{\circ} \mathrm{C}$ until use. Atopy status was defined as one or more positive reactions on skin prick tests with 55 common aeroallergens (Bencard, Bradford, UK). ${ }^{9}$ All of the study subjects provided written informed consent. The study was approved by the Institutional Review Board of Ajou University Medical Center.

\section{Detection of serum-specific IgG and sIgE antibodies to TDI- human serum albumin conjugate by enzyme-linked immunosorbent assay \\ Vapor-type TDI-human serum albumin (TDI-HSA) and mock-HSA conjugates were kindly provided by Dr Adam Wisnewski (Yale University, New Haven, CT, USA). Serum-specific IgG (sIgG) and serum-specific (sIgE) antibodies to TDI-HSA were detected using a homemade enzyme-linked immunosorbent assay (ELISA), as pre- viously described. ${ }^{9,10}$}

\section{ELISA to measure serum levels of FLCN and interleukin-8} Commercial ELISA kits were used to measure the serum levels of FLCN (CUSABIO Biotech, Wuhan, Hubei Province, China) and interleukin-8 (IL-8) (Endogen, Woburn, MA, USA), as well as the levels in cell culture supernatants, following the manufacturer's protocols.

\section{Isolation of peripheral blood neutrophils}

Blood samples were collected from healthy donors into BD Vacutainer tubes containing acid citrate dextrose solution (BD Biosciences, Franklin Lakes, NJ, USA), stored at room temperature (RT), and were processed within $2 \mathrm{~h}$ of collection. Peripheral blood neutrophils (PBNs) were isolated by gradient centrifugation on Lymphoprep solution (Axis-Shield, Oslo, Norway), followed by sedimentation in Hank's balanced salt solution buffer containing 2\% dextran (Polysciences, Warrington, PA, USA), as previously described. ${ }^{11}$ Cell viability ( $>98 \%$ ) was assessed by trypan blue staining. Cell purity (>95\%) was assessed by hematoxylin and eosin (H\&E) staining and flow cytometry using CD68 and CD11b expression.

\section{HAEC culture and treatment}

A human lung carcinoma cell line (A549) was purchased from the American Type Culture Collection (Manassas, VA, USA) and cultured in RPMI-1640 medium supplemented with $10 \%$ fetal bovine serum, $100 \mathrm{U} \mathrm{ml}^{-1}$ penicillin $\mathrm{G}$ sodium and $100 \mu \mathrm{g} \mathrm{ml}^{-1}$ streptomycin sulfate (all from Gibco, Grand Island, NY, USA). Cells were maintained at $37^{\circ} \mathrm{C}$ with $5 \% \mathrm{CO}_{2}$ in humidified air. Cells $\left(2 \times 10^{5}\right)$ were seeded onto each well of a 12-well plate (TPP, Trasadingen, Switzerland) and treated with 2-200 $\mu \mathrm{g} \mathrm{ml} \mathrm{TDI-HSA} \mathrm{in} \mathrm{serum-free} \mathrm{RMPI-1640} \mathrm{med-}$ ium. Mock-HSA conjugate was used as a control (data not shown). In co-culture experiments, different numbers of PBNs were added onto A549 cells in serum-free medium. After a $24 \mathrm{~h}$ incubation, the supernatant was collected, and the cells were lysed in a radioimmunoprecipitation assay buffer and stored at $-70^{\circ} \mathrm{C}$ for further experiments.

\section{Western blotting to detect FLCN}

The proteins $(30 \mu \mathrm{g})$ from A549 cell lysates were loaded onto $10 \%$ SDS-polyacrylamide gels and transferred to polyvinylidene difluoride membranes (Bio-Rad, Hercules, CA, USA). After blocking in 5\% skim milk (Sigma, St Louis, MO, USA) in PBS containing 0.05\% Tween 20 (PBS-T) for $1 \mathrm{~h}$ at RT, the membranes were incubated with rabbit anti-human FLCN antibody (Cell Signaling, Minneapolis, MN, USA) overnight at $4{ }^{\circ} \mathrm{C}$. Then the membranes were washed three times with PBS-T for $10 \mathrm{~min}$ each, and incubated with the appropriate secondary antibody for $1 \mathrm{~h}$ at RT. The membranes were blotted with anti-beta actin as a loading control. Signals were detected using ECL Plus Western Blotting Detection Reagents (GE Healthcare, Little Chalfont, UK). The intensity of bands was analyzed using a gel doc system (Bio-Rad).

\section{Statistical analysis}

The serum levels of FLCN in the study subjects were log-transformed before statistical analysis to establish a normal distribution. Data for continuous variables were compared using the Student's $t$-test or Mann-Whitney $U$-test; Pearson's $\chi^{2}$ or Fisher's exact tests were used for categorical variables. Statistical correlations were analyzed using Pearson's coefficient or Spearman's rank coefficient. All of the statistical analyses were performed with SPSS ver. 22.0 (SPSS, Chicago, IL, USA). $P$ values $<0.05$ were considered statistically significant. GraphPad Prism 5.0 (GraphPad, San Diego, CA, USA) was used for graphs, with values presented as the mean \pm standard deviation (s.d.) of at least three independent experiments.

\section{RESULTS}

\section{Clinical characteristic of the study subjects}

Females predominated in the NOA $(60.0 \%)$ and NC $(60.6 \%)$ groups, whereas males predominated in the TDI-OA $(69.9 \%)$ and AEC (68.1\%) groups (Table 1). The duration of TDI exposure of TDI-OA patients $(6.19 \pm 4.3$ years $)$ was significantly shorter than that of AEC group (12.05 \pm 8.2 years, $P=0.003)$. The prevalence of sIgG and sIgE antibodies against TDI-HSA in the TDI-OA group was significantly higher than that in the AEC group (23.8\% vs $5.9 \%$ and $23.5 \%$ vs $1.7 \%$, respectively; both $P<0.001)$. No differences in serum levels of total IgE or asthma duration were observed between the TDI$\mathrm{OA}$ and NOA groups.

\section{Elevated serum levels of FLCN in TDI-exposed subjects and NOA patients}

We log-transformed the serum level of FLCN to correct its skewed distribution. Serum levels of FLCN (Figure 1a) of the TDI-OA $(1.57 \pm 0.31)$, AEC $(1.64 \pm 0.31)$ and NOA $(1.50 \pm 0.24)$ groups were all significantly higher than those of the NC group $(1.31 \pm 0.21$, all $P<0.001)$. The serum levels of FLCN in both the TDI-OA and AEC groups were significantly higher than those in the NOA group $(P=0.038$ and $P<0.001$, respectively). We classified the study subjects into two groups with high (FLCN-high) and low (FLCNlow) serum levels of FLCN using a cutoff value (1.75) calculated as the mean plus two s.d.'s of the serum level of 
Table 1 Clinical characteristic of the study subjects

\begin{tabular}{|c|c|c|c|c|c|c|c|c|}
\hline & \multirow[b]{2}{*}{$\begin{array}{l}\text { TDI-OA } \\
(\mathrm{n}=93)\end{array}$} & \multirow[b]{2}{*}{$A E C(\mathrm{n}=119)$} & \multirow[b]{2}{*}{ NOA $(\mathrm{n}=200)$} & \multirow[b]{2}{*}{$N C(\mathrm{n}=71)$} & \multicolumn{4}{|c|}{ P-value } \\
\hline & & & & & $\begin{array}{c}T D I-O A \text { vs } \\
A E C\end{array}$ & $\begin{array}{c}\text { TDI-OA vs } \\
\text { NOA }\end{array}$ & $\begin{array}{c}\text { TDI-OA vs } \\
N C\end{array}$ & $\begin{array}{c}A E C \text { vs } \\
N C\end{array}$ \\
\hline Age $^{a}$ & $42.8 \pm 10.5$ & $40.57 \pm 8.7$ & $44.66 \pm 15.8$ & $39.75 \pm 14.0$ & 0.122 & 0.280 & 0.073 & 0.366 \\
\hline Sex $(\text { female })^{b}$ & $28(30.1)$ & 38 (31.9) & $120(60.0)$ & $43(60.6)$ & 0.776 & $<0.001$ & $<0.001$ & $<0.001$ \\
\hline $\mathrm{MchPC}_{20}\left(\mathrm{mg} \mathrm{ml}^{-1}\right)^{\mathrm{a}}$ & $7.04 \pm 13.39$ & $7.94 \pm 9.35$ & $12.42 \pm 12.04$ & NA & 0.266 & $<0.001$ & NA & NA \\
\hline Serum total IgE $\left(I U ~ I^{-1}\right)^{a}$ & $327.2 \pm 497.9$ & $231.05 \pm 618.9$ & $409.75 \pm 719.16$ & NA & 0.097 & 0.294 & NA & NA \\
\hline TDI exposure duration (years) & $6.19 \pm 4.3$ & $12.05 \pm 8.2$ & NA & NA & 0.003 & NA & NA & NA \\
\hline Asthma duration (years) ${ }^{a}$ & $6.68 \pm 4.11$ & NA & $7.2 \pm 6.58$ & NA & NA & 0.619 & NA & NA \\
\hline slgG to TDI-HSA ${ }^{b}$ & 19/80 (23.8) & 7/119 (5.9) & NA & NA & $<0.001$ & NA & NA & NA \\
\hline
\end{tabular}

Abbreviations: AEC, asymptomatic TDI-exposed control; FEV1, forced expiratory volume in $1 \mathrm{~s}$; MchPC20, concentration of methacholine required to produce a $20 \%$ decrease in FEV1; NA, not available; NC, normal healthy control; NOA, non-occupational adult asthma; slgG and slgE, serum-specific IgG and IgE antibody; TDI-HSA, toluene diisocyanate-human serum albumin conjugate; TDI-OA, TDI-induced occupational asthma.

aData are shown as the mean \pm s.d., $P$-value obtained with the Mann-Whitney U-test.

bData are shown as the prevalence (\%), $P$-value obtained with the $\chi^{2}$ test.

Bold numbers indicate statistically significant values.
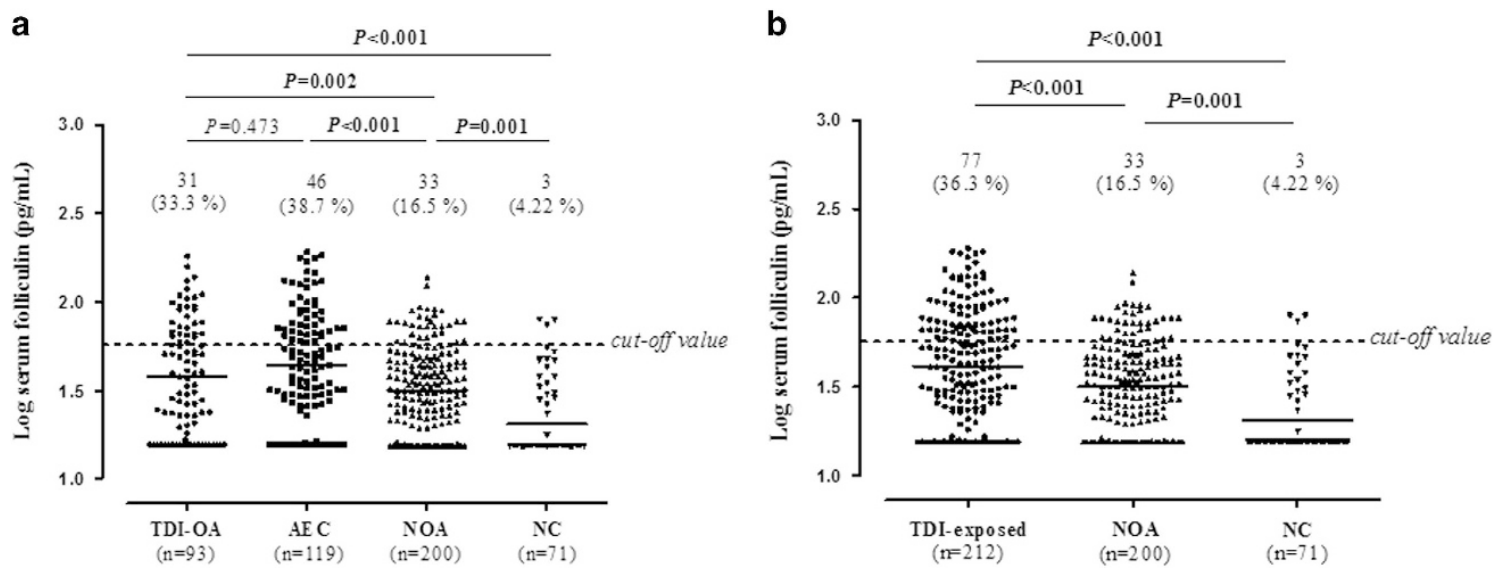

Figure 1 Comparison of serum FLCN levels among the four study groups (a) and among the TDI-exposed subjects (including TDI-OA and AEC groups), NOA and NC groups (b). Dot plots show the log-transformed serum FLCN level. Horizontal dashed lines indicate the cutoff value, and horizontal solid lines in the middle of the dot plots indicate the mean value of each group. The $P$-value was obtained using Fisher's exact test to compare the prevalence of subjects with high serum FLCN levels (\%) among the indicated groups. AEC, asymptomatic TDI-exposed controls; FLCN, folliculin; NC, normal healthy controls; NOA, non-occupational adult asthma; TDI-OA, TDI-induced occupational asthma.

FLCN of the NC group. The prevalence of FLCN-high subjects in the TDI-OA (33.3\%) and AEC (38.7\%) groups did not significantly differ $(P=0.473)$, whereas both were significantly higher than the NOA group $(16.5 \%, P=0.002$ vs TDI-OA and $P<0.001$ vs AEC). The prevalence of FLCNhigh subjects in the NC group (4.2\%) was significantly lower than that in the other three groups $(P<0.001$ for all). Consequently, TDI-exposed subjects (including TDI-OA and AEC subjects) had significantly higher serum levels of FLCN $(1.61 \pm 0.31)$ and significantly higher prevalence of FLCNhigh subjects $(36.3 \%)$ than the NOA and NC groups (all $P<0.001$, Figure 1b).

\section{Associations of serum levels of FLCN with clinical parameters}

We observed that the serum levels of FLCN were positively correlated with TDI-exposure duration $(r=0.251, P=0.027$, Figure $2 \mathrm{a}$ ), but were negatively correlated with asthma duration of the TDI-OA patients $(r=-0.329, P=0.029$, Figure $2 b)$. Nevertheless, no significant correlation between serum levels of FLCN and asthma duration of NOA group was noted $(r=-0.182, P=0.295)$. The TDI-exposed FLCN-high subjects were also significantly older $(P=0.029)$ and had higher serum levels of total $\operatorname{IgE}(P=0.021)$ than the FLCN-low subgroup (Table 2). No associations of serum levels of FLCN with the 

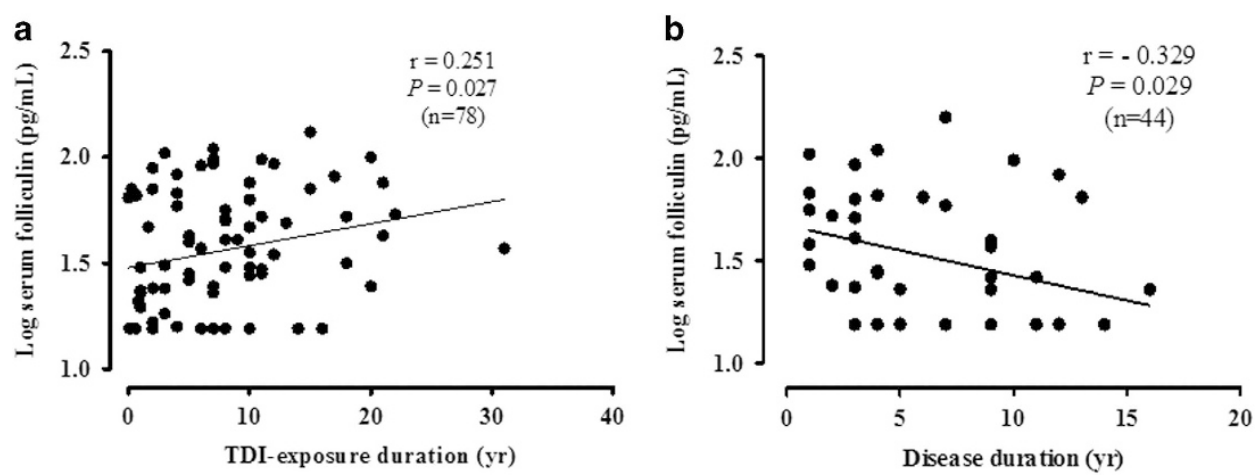

Figure 2 Correlation of serum FLCN level with TDI exposure duration in TDI-exposed subjects (a) and disease duration of TDI-OA patients (b). Spearman's rank coefficient was applied for the test. FLCN, folliculin; TDI, toluene diisocyanate; TDI-OA, TDI-induced occupational asthma.

Table 2 Comparison of the clinical and immunological features according to the level of serum FLCN in TDI-exposed subjects

\begin{tabular}{|c|c|c|c|}
\hline & \multicolumn{2}{|c|}{ Serum FLCN level } & \multirow[b]{2}{*}{ P-valu } \\
\hline & High $(n=77)$ & Low $(n=135)$ & \\
\hline Age (years) ${ }^{a}$ & $43.55 \pm 9.75 / 75$ & $40.38 \pm 9.24 / 133$ & 0.029 \\
\hline Atopy ${ }^{b}$ & $16 / 24(66.7)$ & $24 / 54(44.4)$ & 0.088 \\
\hline Sex (female $)^{b}$ & 20/77 (26.0) & $46 / 135(34.1)$ & 0.280 \\
\hline Asthma duration (years) ${ }^{\mathrm{a}}$ & $5.92 \pm 4.03 / 12$ & $6.97 \pm 4.16 / 32$ & 0.524 \\
\hline FEV1 (\% predicted $)^{a}$ & $81.06 \pm 15.85 / 22$ & $87.79 \pm 23.49 / 47$ & 0.168 \\
\hline MchPC20 (mg ml-1)a & $6.27 \pm 11.76 / 23$ & $7.53 \pm 13.36 / 56$ & 0.791 \\
\hline $\begin{array}{l}\text { Serum total IgE } \\
\left(\mathrm{IU} \mathrm{ml^{-1 }}\right)^{\mathrm{a}}\end{array}$ & $410.5 \pm 723.9 / 40$ & $241.3 \pm 401.2 / 78$ & 0.021 \\
\hline Serum slgG to TDI-HSA & $6 / 71(8.5)$ & 20/128 (15.6) & 0.190 \\
\hline Serum slgE to TDI-HSA & $8 / 72(11.1)$ & $13 / 128(10.2)$ & 0.815 \\
\hline Serum IL-8 (pg ml-1 $)^{a}$ & $48.06 \pm 72.89 / 69$ & $58.9 \pm 139.1 / 127$ & 0.777 \\
\hline
\end{tabular}

Abbreviations: FEV1, forced expiratory volume in $1 \mathrm{~s}$; IL-8, interleukin 8;

MchPC20, concentration of methacholine required to produce a $20 \%$ decrease in

FEV1; slgG and slgE, serum-specific IgG and IgE antibody; TDI-HSA, toluene diisocyanate-human serum albumin conjugate

aData are shown as the mean \pm s.d., $P$-value obtained with the Mann-Whitney U-test.

bData are shown as the prevalence (\%), $P$-value obtained with the $\chi^{2}$ test. Bold numbers indicate statistically significant values.

prevalence of serum sIgE or sIgG to TDI-HSA or serum levels of IL-8 were observed.

Effect of TDI exposure on FLCN production from HAECs When A549 cells were treated with $2-200 \mu \mathrm{g} \mathrm{ml}^{-1}$ TDI-HSA conjugate, TDI-HSA dose-dependently increased both the intracellular expression (Figure 3a) and extracellular release (Figure 3b) of FLCN compared to mock-HSA treatment. TDI-HSA also induced IL-8 production from A549 cells in a dose-dependent manner (Figure 3c), which was strongly correlated with the FLCN level released from those cells $(r=0.779, P=0.003$, Figure $3 \mathrm{~d})$. TDI-HSA did not affect A549 cell viability at the tested concentrations (Figure 3e).
Neutrophils induce FLCN production from A549 cells

Neutrophilic airway inflammation contributes to TDI-OA development. $^{2}$ Because TDI-HSA induced A549 cells to produce IL-8, a strong neutrophil chemoattractant, we evaluated the role of neutrophils in FLCN production by HAECs. A549 cells were co-cultured with $\mathrm{PBNs}$ at different cell number ratios. We observed that $\mathrm{PBNs}$ significantly increased both the intracellular expression (Figure 4a) and extracellular release (Figure 4b) of FLCN from A549 cells in a dose-dependent manner.

\section{DISCUSSION}

Toluene diisocyanate exposure directly damaged HAECs and activated their inflammatory response, leading to airway epithelium impairment and the infiltration of inflammatory cells into the airways. ${ }^{2}$ FLCN is a protein expressed by airway epithelial cells that regulates the survival and adherence of alveolar epithelial cells. ${ }^{7}$ Recently, FLCN has been detected in the bronchial alveolar lavage fluid from asthmatic patients, suggesting a role in asthma. ${ }^{12}$ Nevertheless, the function of FLCN in asthma and its involvement in TDI-OA have not been studied. This is the first study to describe increased serum levels of FLCN in TDI-exposed workers, including TDI-OA and AEC groups, as well as NOA patients compared to normal healthy subjects. We also found that TDI exposure induced the concomitant release of FLCN and IL-8 from A549 cells, which was also induced by co-culture of A549 cells with PBNs. These findings suggest the involvement of FLCN in airway inflammation in TDI-exposed subjects.

A previous study found increased FLCN secretion from compressed human bronchial epithelial cells, ${ }^{12}$ suggesting that FLCN is released from HAECs in asthmatic patients. Consistently, we found higher serum FLCN levels in both TDI-OA and NOA patients compared to healthy subjects, implicating FLCN in the pathogenesis of asthma. We also observed higher production of serum FLCN in TDI-OA and AEC subjects compared to the NOA and NC groups, as well as a positive correlation of serum FLCN levels with TDI-exposure duration, but not with the asthma duration of NOA patients. These findings suggest that TDI is a stronger inducer of FLCN 
a

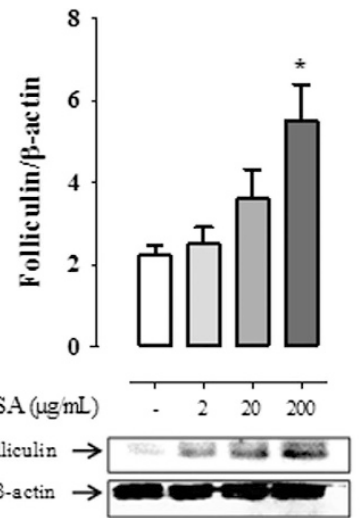

b
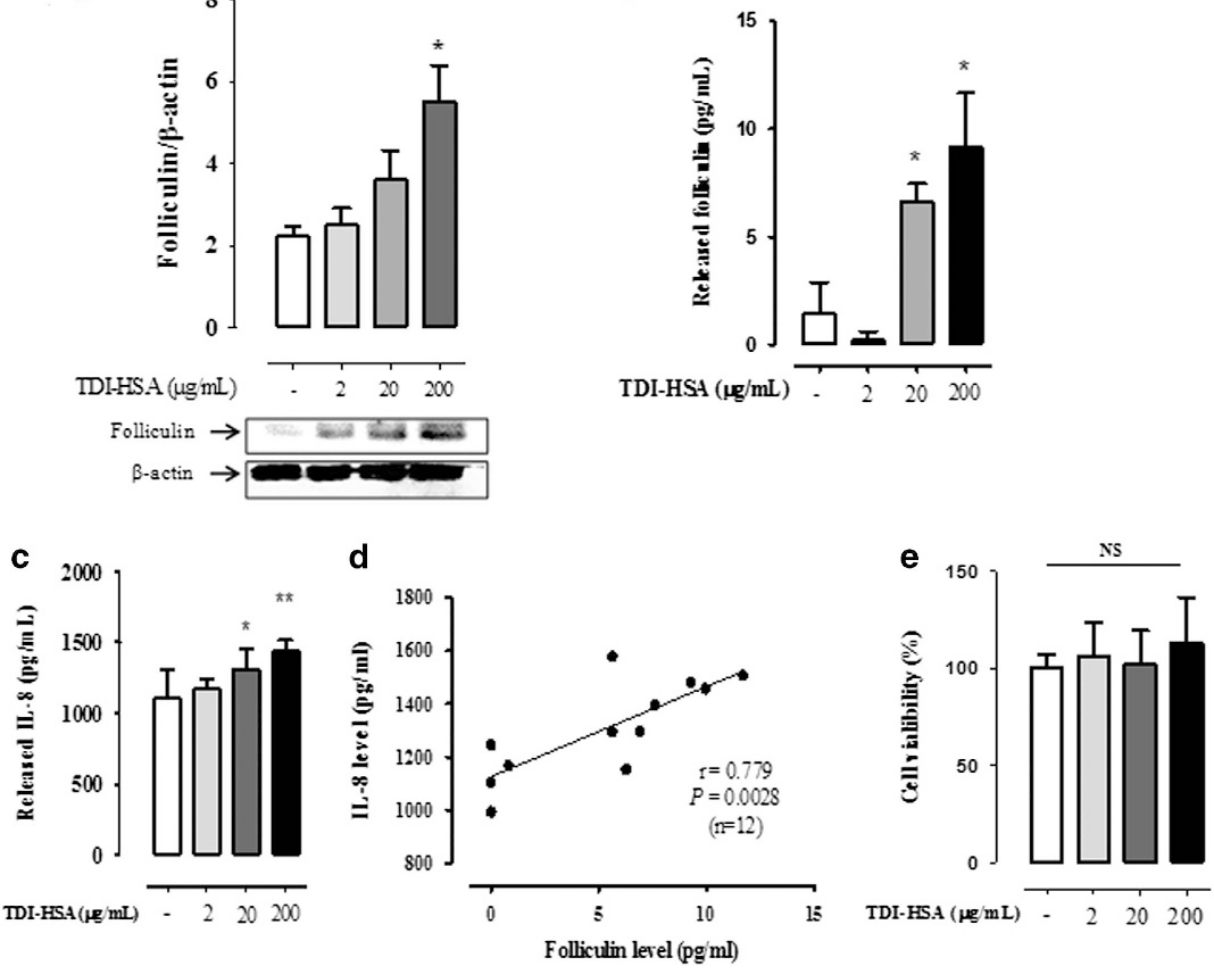

Figure 3 TDI-HSA conjugate-induced FLCN and IL-8 production from A549 cells. A549 cells were treated with TDI-HSA or mock-HSA (negative control) for $24 \mathrm{~h}$. (a) The intracellular expression of FLCN was evaluated by western blotting. The levels of FLCN (b) and IL-8 (c) released to the culture supernatants were evaluated by ELISA. (d) Correlation of FLCN and IL- 8 levels in the culture supernatants. (e) Effect of TDI-HSA conjugate on A549 cell viability was assessed using a Cell Counting Kit 8 . ${ }^{*} P<0.05,{ }^{*} P<0.01$ obtained by oneway ANOVA with Bonferroni's post hoc test. FLCN, folliculin; HAS, human serum albumin; IL, interleukin; NS, not significant.

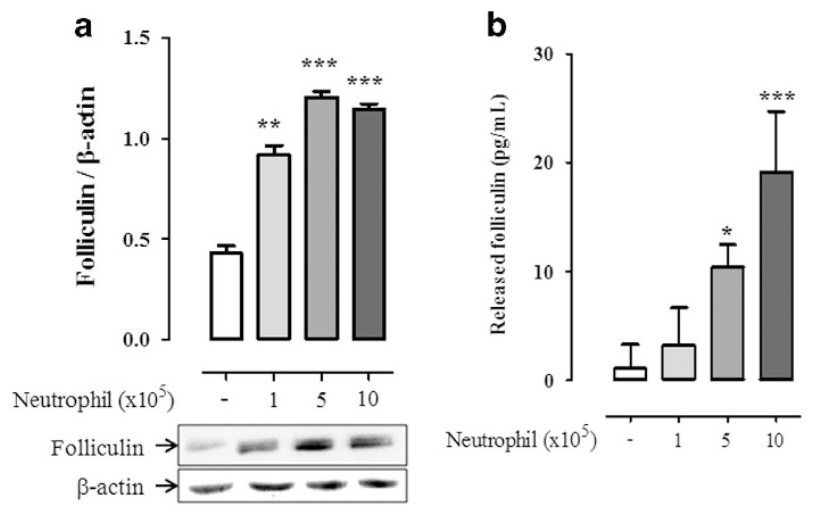

Figure 4 Peripheral blood neutrophils-induced FLCN expression and release from A549 cells. A549 cells $\left(2 \times 10^{5}\right.$ cells) were cocultured with peripheral blood neutrophils for $24 \mathrm{~h}$. (a) Intracellular FLCN expression was evaluated by western blotting. (b) The levels of FLCN released to the culture supernatants were measured by ELISA. ${ }^{*} P<0.05,{ }^{* *} P<0.01,{ }^{* *} P<0.001$ vs control, obtained by one-way ANOVA with Bonferroni's post hoc test. FLCN, folliculin.

production. The role of FLCN in TDI-OA and asthma has not been investigated, although several studies have suggested that FLCN has a protective function maintaining the integrity and survival of HAECs. ${ }^{7}, 13$ Moreover, serum levels of FLCN were negatively correlated with disease duration of the TDI-OA patients, which suggests that long-term airway epithelial cell damage induced by TDI could lead to the reduced production of FLCN. Overall, we speculate that increased expression or secretion of FLCN upon TDI exposure is not only a consequent response but also a protective mechanism against TDI-induced airway damage. The function of FLCN in TDI-OA and NOA remains to be investigated.

The consequences of the aging process have been implicated in innate immune responses, including HAEC dysfunction and increased neutrophils infiltrating into airways of asthmatics. ${ }^{14} \mathrm{We}$ observed that TDI-exposed subjects who had high serum levels of FLCN were older than FLCN-low subjects, suggesting that the aging process affects FLCN production from HAECs. Moreover, airway epithelium is a barrier to the infiltration of allergens into the subepithelial layers where they interact with antigenpresenting cells, which could regulate allergen-specific antibody production. ${ }^{15}$ Therefore, the association of serum levels of FLCN and total serum levels of IgE observed in this study may be an epiphenomenon that resulted from airway damage and increased airway permeability induced by TDI exposure.

To validate our hypothesis that TDI exposure induces FLCN production from HAECs, we treated A549 cells with TDI-HSA conjugate, which dose-dependently increased both the intracellular expression and extracellular release of FLCN. Moreover, the TDI-HSA conjugate stimulated A549 cells to produce IL-8, which could be considered an activation maker of HAECs. ${ }^{9}, 16$ Interestingly, the extracellular FLCN levels strongly 
correlated with the IL-8 levels. These findings suggest that TDI exposure activates HAECs to produce FLCN, explaining the elevated serum FLCN levels in TDI-exposed subjects. However, the increased FLCN level in NOA patients could be a consequence of bronchial contraction, ${ }^{12}$ whereas that in TDIexposed subjects is induced by direct stimulatory effects of TDI. The mechanisms underlying the TDI-induced FLCN expression need further investigation.

Previously, we reported higher numbers of neutrophils infiltrating the airways of TDI-OA patients compared to AEC and NOA subjects, ${ }^{17}$ suggesting the contribution of neutrophils to the development of airway inflammation in TDI-OA. Moreover, we consistently found that TDI exposure stimulates HAECs to produce IL-8, a well-known neutrophil chemoattractant. $^{2,18}$ Consequently, we elucidated the role of neutrophils in FLCN production from HAECs in this study. Co-culture with PBNs significantly increased FLCN expression and release from A549 cells, suggesting that TDI-induced neutrophil infiltration of the airway enhances the production of FLCN by HAECs. However, we did not find any correlation between serum levels of FLCN and sputum neutrophil count or serum levels of IL-8 in TDI-exposed subjects. Perhaps FLCN production in TDI-exposed subjects is also affected by various factors that participate in the airway inflammation process.

In addition, we observed the increased serum FLCN level from TDI-exposed subjects regardless the development of TDI-OA. It is known that the development of TDI-OA depends on various mechanisms, including immunologic, non-immunologic and genetic molecular mechanisms. ${ }^{19}$ We speculate that FLCN is not a determining factor, but involved in TDI-OA by interacting with other factors, which needs to be investigated further.

In conclusion, TDI exposure and TDI-induced neutrophil recruitment into the airways directly activates HAECs and stimulates them to produce FLCN, could be involved in airway inflammation in workers exposed to TDI.

\section{CONFLICT OF INTEREST}

The authors declare no conflict of interest.

\section{ACKNOWLEDGEMENTS}

This study was supported by a grant from the Korean Health Technology R\&D Project, Ministry of Health \& Welfare, Republic of Korea (H14C2628).

\section{PUBLISHER'S NOTE}

Springer Nature remains neutral with regard to jurisdictional claims in published maps and institutional affiliations.

1 Lee SH, Jang AS, Kwon JH, Park SK, Won JH, Park CS. Mesenchymal stem cell transfer suppresses airway remodeling in a toluene diisocyanate-induced murine asthma model. Allergy Asthma Immunol Res 2011; 3: 205-211.

2 Shin YS, Kim MA, Pham LD, Park HS. Cells and mediators in diisocyanateinduced occupational asthma. Curr Opin Allergy Clin Immunol 2013; 13: 125-131.
3 Fajt ML, Wenzel SE. Development of new therapies for severe asthma. Allergy Asthma Immunol Res 2017; 9: 3-14.

4 Luijten MN, Basten SG, Claessens T, Vernooij M, Scott CL, Janssen R et al. Birt-Hogg-Dubé syndrome is a novel ciliopathy. Hum Mol Genet 2013; 22: 4383-4397.

5 Nickerson ML, Warren MB, Toro JR, Matrosova V, Glenn G, Turner ML et al. Mutations in a novel gene lead to kidney tumors, lung wall defects, and benign tumors of the hair follicle in patients with the Birt-Hogg-Dube syndrome. Cancer Cell 2002; 2: 157-164.

6 Sundaram S, Tasker A, Morrell N. Familial spontaneous pneumothorax and lung cysts due to a Folliculin exon 10 mutation. Eur Respir J 2009; 33: 1510-1512.

7 Goncharova EA, Goncharov DA, James ML, Atochina-Vasserman EN, Stepanova V, Hong S-B et al. Folliculin controls lung alveolar enlargement and epithelial cell survival through E-cadherin, LKB1, and AMPK. Cell Rep 2014; 7: 412-423.

8 Hoshika Y, Takahashi F, Togo S, Hashimoto M, Nara T, Kobayashi T et al. Haploinsufficiency of the folliculin gene leads to impaired functions of lung fibroblasts in patients with Birt-Hogg-Dubé syndrome. Physiol Rep 2016; 4: e13025.

9 Pham LD, Kim M-A, Yoon MG, Lee SI, Shin YS, Park HS. Serum specific IgG response to toluene diisocyanate-tissue transglutaminase conjugate in toluene diisocyanate-induced occupational asthmatics. Ann Allergy Asthma Immunol 2014; 113: 48-54.

10 Choi JH, Nahm DH, Kim SH, Kim YS, Suh CH, Park HS et al. Increased levels of IgG to cytokeratin 19 in sera of patients with toluene diisocyanateinduced asthma. Ann Allergy Asthma Immunol 2004; 93: 293-298.

11 Pham DL, Ban GY, Kim SH, Shin YS, Ye YM, Chwae YJ et al. Neutrophil autophagy and extracellular DNA traps contribute to airway inflammation in severe asthma. Clin Exp Allergy 2017; 47: 57-70.

12 Qazvini NT, Park C, Mitchel J, Kim J, Butler JP, Israel E et al. Compression of airway epithelium by bronchospasm causes monolayer unjamming, fluidization, and exosomal folliculin release. Am J Respir Crit Care Med 2014; 189: A3665.

13 Khabibullin D, Medvetz DA, Pinilla M, Hariharan V, Li C, Hergrueter A et al. Folliculin regulates cell-cell adhesion, AMPK, and mTORC1 in a cell-typespecific manner in lung-derived cells. Physiol Rep 2014; 2: e12107.

14 Busse PJ, Mathur SK. Age-related changes in immune function: Effect on airway inflammation. J Allergy Clin Immunol 2010; 126: 690-699.

15 Holgate ST, Lackie P, Wilson S, Roche W, Davies D. Bronchial epithelium as a key regulator of airway allergen sensitization and remodeling in asthma. Am J Respir Crit Care Med 2000; 162: S113-S117.

$16 \mathrm{Kim} \mathrm{JH}$, Yoon MG, Seo DH, Kim BS, Ban GY, Ye YM et al. Detection of allergen specific antibodies from nasal secretion of allergic rhinitis patients. Allergy Asthma Immunol Res 2016; 8: 329-337.

17 Park H, Hwang S, Nahm D, Yim H. Immunohistochemical characterization of the cellular infiltrate in airway mucosa of toluene diisocyanate (TDI)induced asthma: comparison with allergic asthma. J Korean Med Sci 1998; 13: $21-26$

18 Kim BG, Lee PH, Lee SH, Kim YE, Shin MY, Kang Y et al. Long-term effects of diesel exhaust particles on airway inflammation and emodeling in a mouse model. Allergy Asthma Immunol Res 2016; 8: 246-256.

19 Palikhe NS, Kim JH, Park HS. Biomarkers predicting isocyanateinduced asthma. Allergy Asthma Immunol Res 2011; 3: 21-26.

This work is licensed under a Creative Commons Attribution-NonCommercial-ShareAlike

4.0 International License. The images or other third party material in this article are included in the article's Creative Commons license, unless indicated otherwise in the credit line; if the material is not included under the Creative Commons license, users will need to obtain permission from the license holder to reproduce the material. To view a copy of this license, visit http:// creativecommons.org/licenses/by-nc-sa/4.0/

(C) The Author(s) 2017 